\title{
A stressful life (or death): Combinatorial proteotoxic approaches to cancer-selective therapeutic vulnerability
}

\author{
Paul Workman ${ }^{1}$ and Faith E. Davies ${ }^{2}$ \\ ${ }^{1}$ Signal Transduction and Molecular Pharmacology Team, Cancer Research UK Cancer Therapeutics Unit, Division of Cancer \\ Therapeutics, The Institute of Cancer Research, Haddow Laboratories, 15 Cotswold Road, Sutton, Surrey SM2 5NG UK \\ 2 Myeloma Targeted Treatment Team, Divisions of Molecular Pathology, Clinical Studies \& Cancer Therapeutics, Institute of \\ Cancer Research, Brookes Lawley Building, 15 Cotswold Road, Sutton, Surrey SM2 5NG UK
}

Correspondence to: Paul Workman, email: Paul.workman@icr.ac.uk

Faith Davies, email: Faith.davies@icr.ac.uk

Commentary on: Neznanov, N., Komarov, A., Neznanova, L., Stanhope-Baker, P., \& Gudkov, A. (2011). Proteotoxic stress targeted therapy (PST): induction of protein misfolding enhances the antitumor effect of the proteasome inhibitor bortezomib. Oncotarget, 2(3), 209-221.

Received: April 21, 2011,

Accepted: April 21, 2011,

Published: April 21, 2011

Copyright: @ Workman et al. This is an open-access article distributed under the terms of the Creative Commons Attribution License, which permits unrestricted use, distribution, and reproduction in any medium, provided the original author and source are credited.

Maintaining protein homeostasis within a cell is vital. Recent studies have suggested that therapeutically manipulating intracellular protein handling pathways in cancer cells perturbs protein homeostasis and results in the delivery of a novel apoptotic signal.

A new paper in Oncotarget by Neznanov et al [1] reinforces the potential of proteotoxic stress-targeted therapy and in particular demonstrates the ability of combinatorial approaches to enhance the antitumor effects of the proteasome inhibitor bortezomib by induction of protein misfolding using hyperthermia or the antibiotic puromycin. In particular, the new results illustrate the therapeutic potential of combining non-toxic doses of puromycin with bortezomib in a mouse model of multiple myeloma.

There are a number of potential ways of perturbing protein homeostasis: firstly by forcing the apoptotic signal by disturbing protein quality control with the premature degradation of key growth and survival molecules; secondly by inhibiting the degradation of proteins resulting in a build up of unwanted proteins; or finally by interfering with key protein folding pathways resulting in the build-up of misfolded proteins. The end result of each of these processes is programmed cell death. Crucially, malignant cells are more susceptible to killing through the manipulation of proteostasis, resulting in a cancerselective vulnerability.

The build up of proteins that have failed to fold correctly results in the presence of non-functional proteins, a tendency toward protein aggregation and impaired cellular function and is referred to as proteotoxic stress (PS). A cell placed under such stress has two possible physiological responses. Initially it will resist death whilst attempts at correct protein folding are carried out. However, if this fails then an apoptotic signal is delivered. Two highly conserved systems are in place to combat PS - the unfolded protein response (UPR) and the heat shock response (HSR). Both systems act as quality control processes ensuring the correct folding and 3D conformation for functionally active proteins. The UPR senses unfolded native proteins within the endoplasmic reticulum (ER) and ensures their correct folding, processing, export or degradation. Activation of the UPR results in a bias of protein translation towards the synthesis of chaperone proteins involved in protein folding within the ER, an increase in disposal of misfolded proteins via the ubiquitin proteasome pathway, and the delivery of a survival signal. If the build-up of misfolded protein is irreversible, the cell undergoes apoptosis $[2,3]$. The HSR is activated by the accumulation of non-native proteins in the cytosol or nucleus. Once activated there is an increase in the synthesis of molecular chaperones that both facilitate protein folding and also suppress protein aggregation. In addition, the heat shock proteins also have a broader anti-apoptotic role mediating both the intrinsic mitochondrial-dependent and extrinsic death receptor-dependent apoptotic pathways [4,5]. The balance, therefore, between the induction of proteotoxic stress and the adaptive UPR and HSR is vital for protein homeostasis and cell survival (Figure 1).

A number of studies have demonstrated that cancer cells have intrinsically high levels of PS. This is a result of the accumulation of misfolded proteins caused by cancer cells surviving within an unfavourable hypoxic microenvironment, as well as an increase in protein misfolding 
resulting from aneuploidy and the expression of mutated or over-abundant oncogenic proteins, especially in highly secretory tumours. The increased level of PS results in a dependence of the cancer cell on both the UPR and HSR to maintain protein homeostasis and allow survival in the stressed malignant state. Studies have demonstrated that cancer cells overexpress heat shock protein family members [4,5] and have high levels of a number of components of the UPR pathway (e.g. XBP1s) [6] which aid cell survival and mediate drug resistance.
Over recent years a number of investigators have attempted to alter the balance between PS, the UPR and the HSR to induce cancer cell death. The clinically most advanced of these approaches involve the induction of PS using either proteasome or heat shock protein 90 (HSP90) inhibition. The ubiquitin proteasome pathway is responsible for the degradation of many key cellular signaling proteins. In multiple myeloma, where the use of proteasome inhibitors is considered standard of care, both in-vitro data and clinical studies have demonstrated that inhibition of the catalytic subunit of the $20 \mathrm{~S}$ proteasome

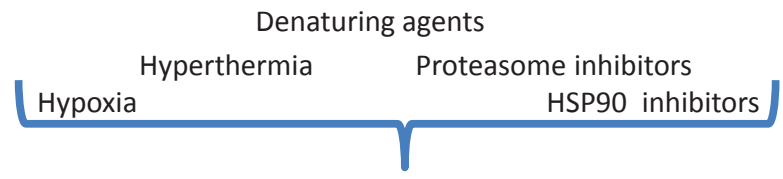

Proteotoxic stress

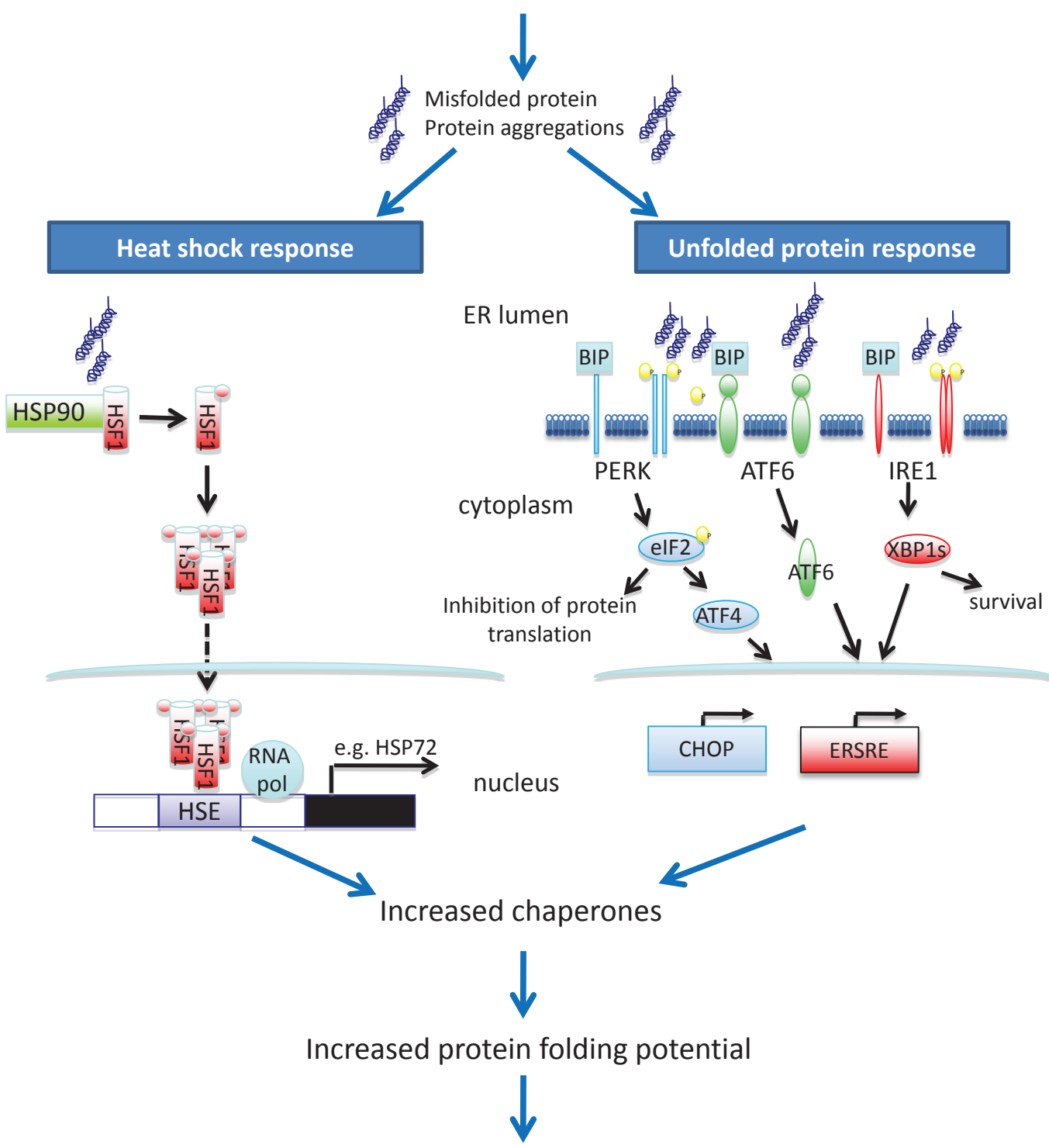

Cell survival

Figure 1: Vulnerable proteotoxic stress pathways for exploitation in cancer-selective combinatorial therapies. 
by bortezomib results in a build up of unwanted proteins, the induction of cellular stress and apoptosis $[7,8]$. Similar results have been seen both in-vitro and in-vivo in solid and hematological malignancies following HSP90 inhibition $[9,10,11]$. HSP90 is a crucial chaperone protein, ensuring correct folding and activation of its clients, which if not correctly folded are targeted for degradation by the proteasome. Many of HSP90's client proteins are key tumor growth and survival signaling molecules such as EGFR, AKT, BRAF, CRAF, CDK4 and mutant p53. HSP90 inhibition results in a decrease in survival molecules, thus sensitizing cells to apoptosis, as well as preventing correct protein folding leading to increased PS.

In addition to inducing PS, a number of investigators have explored approaches aimed at inhibiting the HSR. The heat shock protein 70 family (HSP70) is a major mediator of the HSR. Heat-shock inducible HSP70 (HSP72) plays a key role in protecting cells from stress by reducing stress-induced protein aggregations and thus protecting cells from apoptosis. It also interacts with members of the intrinsic and extrinsic apoptotic pathways and $\mathrm{p} 53$, as well as being a co-chaperone for HSP90. The expression of HSP70 family members is tightly controlled by the transcription factor, heat shock factor 1 (HSF1). In response to stress, including HSP90 inhibition, HSF1 dissociates from the HSP90/HSP72 complex and translocates to the nucleus where it initiates transcription of protective stress response chaperones, reducing damaged protein aggregations and facilitating degradation by the proteasome. The constitutively expressed heat shock cognate 70 (HSC70), another HSP70 family member, also plays a significant anti-apoptotic role. Our own studies have shown that silencing HSC70 or HSP72 alone has no effect on tumor cell death, whereas simultaneously reducing the expression of both of these isoforms induces degradation of HSP90 clients, G1 arrest and apoptosis $[12,13]$. Others have shown similar results with HSF1 silencing $[14,15]$.

A number of groups have explored a combination of the above approaches - targeting PS and HSR simultaneously. Proteasome or HSP90 inhibition results in activation of the HSR via HSF1 driving upregulation of HSP70 family members $[3,10,16]$. Using siRNA we have shown increased susceptibility to HSP90 inhibitors when HSP72 and HSC70 are targeted simultaneously $[12,16]$. Therefore, combinations involving either the proteasome or HSP90 inhibition with HSP70 targeting offers an attractive way to potentiate single agent therapeutic activity.

Neznanov et al present a further approach to manipulating the balance between PS and HSR [1]. Rather than inhibiting the HSR, they dramatically increase PS by both inducing protein misfolding and inhibiting protein degradation via the proteasome, so-called 'enhanced proteotoxic stress'. They demonstrate that the increased level of misfolded proteins, induced by either hyperthermia or puromycin (which works by causing premature termination of translation and accumulation of aborted and incorrectly folded products) in combination with bortezomib, could not be matched by the cancer cell's ability to synthesize chaperones as part of the HSR and thus cell death ensued. The studies indicate that the enhanced proteotoxic death is, at least in part, mediated through $\mathrm{p} 53$. This raises questions as to the effect of p53 status in tumor versus healthy cells on the response to combinatorial treatments proposed. Importantly, however, the synergistic anticancer effect was seen both in-vitro and an in-vivo mouse model of multiple myeloma and thus provides a rationale for attempting such an approach or other PS combinations in the clinical arena.

A wealth of data now supports various tactics to modulate protein homeostasis as an approach to cancerselective therapy. The stressed state of malignant cells leaves them vulnerable to therapeutic strategies that target molecular determinants of the UPR or the HSR or a combination of the two - as orthogonal approaches to exacerbate enhanced PS. Indeed stress phenotypes, including PS, can now be included among the extended hallmarks of cancer - in addition to the Hanahan and Weinberg traits [17]. Furthermore, proteotoxic stress targets can be considered as part of a broader conceptual framework in which non-oncogene targets as well as oncogene targets can be attacked in tumour-selective therapy [17].

\section{ACKNOWLEDGMENTS}

FED is a Cancer Research UK Senior Cancer Fellow and PW is a Cancer Research UK Life Fellow.

\section{CONFLICT OF INTEREST}

The Institute of Cancer Research has a commercial interest in the inhibitors of protein quality control pathways and operates a rewards to inventors scheme.

\section{REFERENCES}

1. Neznanov N, Komarov AP, Neznanova L, Stanhope-Baker P, Gudkov AV. Proteotoxic stress targeted therapy (PSTT): induction of protein misfolding enhances the antitumor effect of the proteasome inhibitor bortezomib. Oncotarget. 2011; 2: 209-21.

2. Davenport EL, Morgan GJ, Davies FE. Untangling the unfolded protein response. Cell Cycle. 2008; 7: 865-9.

3. Davenport EL, Moore HE, Dunlop AS, Sharp SY, Workman P, Morgan GJ, Davies FE. Heat shock protein inhibition is associated with activation of the unfolded protein response (UPR) pathway in myeloma plasma cells. Blood. 2007; 110: 2641-2649.

4. Sharp S, Workman P. Inhibitors of the HSP90 molecular 
chaperone: current status. Advances in Cancer Research. 2006; 95: 323-348.

5. Powers MV, Workman P. Inhibitors of the heat shock response: biology and pharmacology. FEBS Lett. 2007;581:3758-69.

6. Bagratuni T, Wu P, Gonzalez de Castro D, Davenport EL, Dickens NJ, Walker BA, Boyd K, Johnson DC, Gregory W, Morgan GJ, Davies FE. XBP1s levels are implicated in the biology and outcome of myeloma mediating different clinical outcomes to thalidomide-based treatments. Blood. 2010 116(2):250-3

7. Richardson PG, Sonneveld P, Schuster MW, Irwin D, Stadtmauer EA, Facon T, Harousseau JL, Ben-Yehuda D, Lonial S, Goldschmidt H, Reece D, San-Miguel JF, Bladé J, Boccadoro M, Cavenagh J, Dalton WS, Boral AL, Esseltine DL, Porter JB, Schenkein D, Anderson KC. Bortezomib or high-dose dexamethasone for relapsed multiple myeloma. N Engl J Med. 2005; 352:2487-98.

8. Hideshima T, Richardson P, Chauhan D, Palombella VJ, Elliott PJ, Adams J, Anderson KC. The proteasome inhibitor PS-341 inhibits growth, induces apoptosis, and overcomes drug resistance in human multiple myeloma cells. Cancer Res. 2001;61:3071-6.

9. Mitsiades CS, Mitsiades NS, McMullan CJ, Poulaki V, Kung AL, Davies FE, Morgan G, Akiyama M, Shringarpure R, Munshi NC, Richardson PG, Hideshima T, Chauhan D, Gu X, Bailey C, Joseph M, Libermann TA, Rosen NS, Anderson KC. Anti-myeloma activity of heat shock protein-90 inhibition. Blood. 2006;107:1092-100.

10. Pacey S, Wilson RH, Walton M, Eatock MM, Hardcastle A, Zetterlund A, Arkenau HT, Moreno-Farre J, Banerji U, Roels B, Peachey H, Aherne W, de Bono JS, Raynaud F, Workman P, Judson I. A Phase I Study of the Heat Shock Protein 90 Inhibitor Alvespimycin (17-DMAG) Given Intravenously to Patients with Advanced Solid Tumors. Clin Cancer Res. 2011;17:1561-70.

11. Modi S, Stopeck AT, Gordon MS, Mendelson D, Solit DB, Bagatell R, Ma W, Wheler J, Rosen N, Norton L, Cropp GF, Johnson RG, Hannah AL, Hudis CA. Combination of trastuzumab and tanespimycin (17-AAG, KOS-953) is safe and active in trastuzumab-refractory HER-2 overexpressing breast cancer: a phase I dose-escalation study. J Clin Oncol. 2007;25: 5410-7

12. Powers MV, Clarke PA, Workman P. Dual targeting of HSC70 and HSP72 inhibits HSP90 function and induces tumor-specific apoptosis. Cancer Cell. 2008; 14: 250-62.

13. Powers MV, Jones K, Barillari C, Westwood I, van Montfort RL, Workman P. Targeting HSP70: The second potentially druggable heat shock protein and molecular chaperone? Cell Cycle. 2010; 9: 1542 - 1550 .

14. de Billy E, Powers MV, Smith JR, Workman P. Drugging the heat shock factor 1 pathway: exploitation of the critical cancer cell dependence on the guardian of the proteome. Cell Cycle. 2009; 8: 3806-8.
15. Dai C, Whitesell L, Rogers AB, Lindquist S. Heat shock factor 1 is a powerful multifaceted modifier of carcinogenesis. Cell. 2007; 130: 1005-18

16. Davenport EL, Zeisig A, Aronson LI, Moore HE, Hockley S, Gonzalez D, Smith EM, Powers MV, Sharp SY, Workman P, Morgan GJ, Davies FE. Targeting heat shock protein 72 enhances Hsp90 inhibitor-induced apoptosis in myeloma. Leukemia. 2010; 24: 1804-7.

17. Luo J, Solimini NL, Elledge SJ. Principles of cancer therapy: oncogene and non-oncogene addiction. Cell. 2009; 136: 823-37. 